$1981 \mathrm{~b}$ - Chemogeography of Aniba (Lauraceae). Plant Syst. Evol., 137: 281-289.

GOTTLIEB, O.R. \& MORS, W.B.

1959 - Isolation of 5,6-dehydrokawain from Aniba firmula Mez. J. Org. Chem., 24: 17-18.

MASCARENHAS, Y.P. \& GOTTLIEB, O.R.

1977 - Structure of aniba-dimer A isolated from Aniba gardneri. Phytochemistry, 16: 301302.

MORS, W.B.; MAGALHÃES, M.T.; LIMA, O.A.;

GOTTLIEB, O.R.

1962 - Isolamento e síntese de 11-metoxi-iangonina e de 5,6-dehidrometisticina. Anais assoc. brasil. quím., 21: 7-12.
REZENDE, C.M.A. da M.; BÜLOW, M.V. VON; GOTTLIEB, O.R.; PINHO, S.L.V.; ROCHA, A.I. da

1971 - The 2-pyrones of Aniba species. Phytochemistry, 10: 3167-3172.

SANTOS, M.M. dos; MESQUITA, A.A.L.; GOTTLIEB, O.R. 1982 - Estirilpironas de Aniba kappleri. Acta Amazonica, 12: no prelo.

\title{
A Química de Lauráceas Brasileiras. LXX. Estirilpironas de Aniba kappleri
}

(Aceito para publicação em 23/06/82)

\author{
Margarida M. dos Santos \\ Antônio A. L. Mesquita \\ Universidade Federal de Minas Gerais \\ Otto R. Gottlieb \\ Universidade de Sáo Paulo
}

Um estudo botânico assinalou a existência no gênero Aniba (família Lauraceae) de 41 espécies distribuídas em dois grupos morfológicos (Kubitzki, 1982). Estes grupos se caracterizam por uma composição química diversa, as espécies do primeiro grupo contendo neolignanas (Gottlieb, 1977) e as do segundo grupo contendo pironas (Gottlieb, 1972). É interessante assinalar que até hoje nenhuma espécie de Aniba revelou possuir ambos os tipos estruturais (Gottlieb \& Kubitzki, 1981b). A homogeneidade do gênero é indicada assim mesmo pela presença, praticamente em todas as espécies analisadas, de esteres benzílicos dos ácidos benzóico e salicílico e de alcalóides benziltetraidroisoquinolínicos (Ferreira et al., 1980). Este fato possibilita o seu tratamento químiossistemático unificado (Gottlieb, 1980).

Em continuação aos estudos sobre a composição química de lauráceas brasileiras (parte LXIX: Haraguchi et al., 1982), analisamos Aniba kappleri Mez, uma espécie arbórea que ocorre na periferia da Amazônia em direção ao extremo Nordeste da América do Sul. Do ponto de vista morfológico, a espécie foi classificada como pertencente ao segundo grupo acima mencionado e deveria, portanto, conter pironas. O presente trabalho confirma a hipótese indicando a existência de 4-metoxi-6-(3', 4'-metilenodioxi-E-estiril)-2-pirona (1) e de 4-metoxi-6-(3',4'-dimetoxi-E-estiril)-2-pirona (2). Estas substâncias foram obtidas por fracionamento cromatográfico do extrato benzênico do lenho. Do líquido de recristalização de 2, obteve-se um de seus dímeros (3). Se 3 já existisse no extrato, dificilmente teria acompanhado o monómero (2) na passagem pela coluna de gel de sílica. Por isto, é provável que 2 se tenha transformado em 3 , após separação do extrato ou durante a recristalização, por fotodimerização. Uma reação deste tipo já havia sido observada por nós para outra estirilpiro-' na (Gottlieb et al., 1975). A substância 3 deve, portanto, ser considerada um artefato. 
Entre os outros constituintes do extrato encontram-se benzoato de benzila (4), salicilato de benzila (5) e 2,6-diidroxi-benzoato de benzila (6), aiém de sitosterol (7), ácido octacosanóico (8) e rhamnocitrina (7-O-metilkaempferol, 9).

\section{PARTE EXPERIMENTAL}

De um espécimen de A. káppleri do Amapá, identificado por João Murça Pires, coletou este mesmo botânico uma amostra de tronco. Separada a casca, foi sua madeira reduzida a serragem $(2.8 \mathrm{~kg})$ e extraída sucessivamente com benzeno e com etanol pelo químico Mauro Taveira Magalhães. O extrato benzênico $(100 \mathrm{~g})$ foi cromatografado em coluna de gel de sílica $(600 \mathrm{~g})$, coletando-se frações de 120 $\mathrm{ml}$ com $\mathrm{C}_{6} \mathrm{H}_{6}-\mathrm{CHCl}_{3}$ 5:1 e 2:1 (frs. 1-32), 2:1 e $1: 1$ (frs. 33-85), 1:1 (frs. 86-116, 117-127, 128-143, 144-193), 0:1 (frs. 194-382). O resíduo de evaporação das frações 1-32 (49 g) foi separado por cromatografia em coluna seca de gel de sílica em 5 ( $5 \mathrm{~g}), 4(35 \mathrm{~g})$ e $(6 \mathrm{~g})$. O resíduo das frações 86-116 (1.7 g), por recristalização em $\mathrm{MeOH}$, deu a substância 7 (750 mg). O resíduo das frações 117-127 (6 g), por recristalização em EtOH, deu a substância $1(600 \mathrm{mg})$. O resíduo das frações 128-143 (3 g), por recristalização em EtOH, deu a substância 2 (220 $\mathrm{mg}$ ). A solução filtrada foi evaporada e o resíduo, após recristalização em $\mathrm{E}+\mathrm{OH}$, deu a substância $3(270 \mathrm{mg})$. O resíduo das frações 144-193 (5 g) foi recromatografado em coluna de gel de sílica, $\mathrm{CHCl}_{3}$ eluíndo a substância $8(60 \mathrm{mg})$ e $\mathrm{CHCl}_{3}-\mathrm{MeOH}$ 99:1 eluíndo a substância 9 (3 mg). O resíduo das frações 194-382 $(6.5 \mathrm{~g})$ foi lavado com $\mathrm{MeOH}$. A parte insolúvel foi recromatografada em coluna de gel de sílica, $\mathrm{CHCl}_{3}$ eluíndo a substância $9(220 \mathrm{mg})$. O extrato etanólico $(20 \mathrm{~g})$, por um processo de fracionamento análogo, deu quantidades adicionais das substâncias 5,4 e 6 (ao todo 90 mg), 7 (30 mg) e 1 (50 mg).

As identificações das substâncias foram efetuadas por comparação de suas caracterís. ticas, pontos de fusão (exceto para os óleos 4,5 e 6) e espectros no ultravioleta, no infravermelho, de ressonância magnética protônica e de massas, com as características análogas registradas na literatura para 1 , previamente isolada de Aniba heringerii Vattimo (Mors et al., 1962) e de A. panurensis (Meissn.) Mez (Motidome et al., 1982); 2, previamente isolada de A. canelilla (H.B.K.) Mez (Rezende et al., 1971) e de A. panurensis (Motidome et al., 1982); 3, previamente obtida por fotodimerização de 2 (Barth et al., 1967); 4 e 5, previamente isoladas de muitas espécies de Aniba (Gotṫlieb \& Kubitzki, 1981a, 1981b); 6, inédita como substância natural, cuja metilação $\left(\mathrm{Me}_{2} \mathrm{SO}_{4}\right.$, $\mathrm{K}_{2} \mathrm{CO}_{3}, \mathrm{Me}_{2} \mathrm{CO}$ ) deu 2,6-dimetoxibenzoato de benzila, previamente isolado de $\mathrm{A}$. ferrea Kubitzki (Andrade et al., 1980); e 9, inédita para o gênero Aniba, previamente isolada de espécies das famílias Fabaceae, Hippocastanaceae, Rhamnaceae e Zingiberaceae (Gottlieb, 1975).

\section{SumiMARY}

The trunk wood of Aniba kappleri Mez (family Lauraceae) contains besides sitosterol, octacosanoic acid and rhamnocitrin (7-O-methylkaempferol), three esters, namely benzyl benzoate, benzyl 2-hydroxybenzoate and benzyl 2,6-dihydroxybenzoate, and two $\alpha$-pyrones, namely 4-methoxy-6- (E) -(3',4'-methylenedioxystyryl)-2-pyrone and 4-methoxy-6-(E)-(3',4'-dimethoxystyryl)-2pyrone. A dimer of the latter compound, which was obtained additionally, is considered to be an artifact.

\section{Agradecimentos}

Os autores agradecem ao CNPq pelas bolsas concedidas e à FINEP pelo apoio financeiro ao programa de pesquisas.

\section{REFERENCIAS BIBLIOGRÁFICAS}

ANDRADE, C.H.S.; BRAZ FILHO, R.; GOTTLIEB, O.R. 1980 - Neolignans from Aniba ferrea. Phytochemistry, 19: 1191-1194.

BARTH, K.D.; EDWARDS, R.L.; JONES, D.W.; MIR, I. 1967 - The photodimerization of hispidin analogues, a proton magnetic resonance study. J. Chem. Soc., C: 413-419.

FERREIRA, Z.S.; GOTTLIEB, O.R. \& ROQUE, N.F. 1980 - Chemosystematic implications of benzyltetrahydroisoquinolines in Aniba. Biochem. Syst. Ecol., 8: 51-54. 
GOTTLIEB, O.R.

1972 - Chemosystematics of the Lauraceae. Phytochemistry, 11: 1537-1570.

1975 - Flavonols, ps. 296-375. In: Harbone, J.B., Mabry, T.J. \& Mabry, H. (eds.) The Flavonoids. London, Chapman \& Hall.

1977 - Neolignans. Progr. Chem. Org. Nat. Prod., 35: $1-37$.

1980 - Towards a scientific status for micromolecular systematics. Acta Amazonica, 10: 845-862.

GOTTLIEB, O.R. \& KUBITZKI, K.

1981a - Chemogeography of Aniba (Lauraceae). Plant Syst. Evol., 137: 281-289.

1981b-Chemosystematics of Aniba. Biochem. Syst. Ecol., 9: 5-12.

GOTTLIEB, O.R.; Veloso, D.P.; PEREIRA, M.O. da S.

1975 - The photodimerization of 4-methoxy-6-(E)-styryl-2-pyrone. Rev. Latinoamer. Quím., 6: 188-190.
HARAGUCHI, M.; MOTIDOME, M.; YOSHIDA, M.; GOTTLIEB, O.R.

1982 - Neolignans from Ocotea catharinensis. Phytochemistry, no prelo.

KUBITZKI, K.

1982 - Monograph on Aniba. Flora Neotropica, no prelo.

MORS, W.B.; MAGALHÃES, M.T.; LIMA, O.A.;

GOTTLIEB, O.R.

1962 - Isolamento e síntese de 11-metoxi-iangonina e de 5,6-de-hidrometisticina. Anais assoc. brasil. quím., 21: 7-12.

MOTIDOME, M.; GOTTLIEB, O.R.; KUBITZKI, K.

1982 - Styrylpyrones of Aniba panurensis and A. permollis. Acta Amazonica, 12: no prelo.

REZENDE, C.M.A. da M.; BÜLOW, M.V. VON;

GOTTLIEB, O.R.; PINHO, S.L.V.; ROCHA, A.I. da 1971 - The 2-pyrones of Aniba species. Phytochemistry, 10: 3167-3172.

(Aceito para publicação em 07/06/82) 